\title{
- BOOK REVIEWS -
}

\section{The Hidden Psychology of Pain: The Use of Understanding to Heal Chronic Pain}

James Alexander. Balboa Press, Bloomington, IN, 2012, 470 pp., $\$ 40.99$ (paperback), \$7.11 (Kindle)

Alexander states the problem convincingly: Medical and pharmaceutical approaches to chronic pain (defined as lasting for more than three months) have failed. Prescription and illegal drug use is epidemic and frequently results in addictive and lethal side effects. Science and compassionately presented case studies defend the premise that chronic pain is perhaps the body-mind health condition. This timely introduction (Chapters 1 through 7 ) is recommended to all who experience chronic pain or illness - and to professionals who treat chronic pain victims.

The solutions pages (mostly pages 140 and beyond) may provide hope to open-minded clinicians seeking new ideas and to their chronic pain clients. Overall, recommendations for patients are sound: Be medically screened, distinguish between physical and psychogenic contributions, practice self-care (yoga, meditation), and consider how unresolved trauma may cause and maintain chronic pain.

References to specific interventions are more problematic; this section is less orderly, and opinion is sometimes offered as fact in spite of Alexander's claim that "everything in this book is supported by scientific evidence" (p. xv). The author, a clinical health psychologist, implies early on that the vast majority of chronic pain is reduced with accurate information; he also promotes "depth" psychotherapy. Comments about these interventions are consistent with the subtitle ("understanding" causes chronic pain relief) but neither is defended with science, and no specific depth therapies are endorsed. The book would be strengthened if this part were reduced and better clarified.

Alexander highlighted two innovative therapies as having special potential for healing chronic pain: emotional freedom techniques (EFT) and eye movement desensitization and reprocessing (EMDR). Paradoxically, neither of these requires understanding to produce benefit. Case testimonials and clear discussions of the potential of both EFT and EMDR make a good case for further investigation of their value and applicability with chronic pain sufferers. Experienced EFT and EMDR clinicians who treat pain suf- ferers will likely find the chapters generally credible and the case studies moving and familiar.

The science cited, however, is insufficiently convincing. Although controlled studies support both EFT and EMDR for trauma resolution, data are not available to justify Alexander's linking trauma and chronic pain. Hence, in a recent and comprehensive review of treatments for chronic pain (Jensen \& Turk, 2014), neither EFT nor EMDR was mentioned. Well-narrated testimonials, left to speak for themselves, can be persuasive, even inspirational. Equating them with evidence can leave skeptics, especially academics, more skeptical.

The author correctly acknowledges "other factors" that contribute to chronic pain - in addition to unresolved trauma and related psychological causes. In this context, clinicians who work with pain victims may want to supplement the book with training in addictions (not covered), particularly when opiate-based pharmaceuticals and street drugs are involved. In addition, a team approach to treatment (also not covered) would be encouraged. EMDR clinicians may want to study manuals that specify those other factors, including a helpful EMDR approach pioneered by Mark Grant and an especially thorough EMDR protocol for migraine pain sufferers developed by Steven Marcus.

\section{REVIEWED BY JOHN HARTUNG}

\section{Reference}

Jensen, M. P., \& Turk, D. C. (2014). Contributions of psychology to the understanding and treatment of people with chronic pain: Why it matters to ALL psychologists. American Psychologist, 69(2), 105-118.

\section{Healing the Folks Who Live Inside: How EMDR Can Heal Our Inner Gallery of Roles}

Esly Regina Carvalho. Self-published, Brazilian Association of EMDR, Printed in the USA , 2013, 86 pp., $\$ 12.00$ (paperback)

Dr. Carvalho studied with Francine Shapiro in 1997 and was one of the first eye movement desensitization and 
reprocessing (EMDR) trainer of trainers in Latin America. She has long been a trainer of psychodrama, perhaps the first psychotherapy to address ego states and dissociation. Dr. Carvalho skillfully combines the role theory of Jacob Moreno, a peer of Freud, and Carlos Raimundo's Play of Life with EMDR. She writes, "A role is a tangible way of being . . . According to Moreno, the self is born from the roles ... we will spend our life integrating these roles that are constantly forming so that we can acquire a greater and greater sense of an integrated self' (p. 32). The Play of Life uses three mini-stages and Playmobil dolls to discover, understand, and modify aspects of people's relationships (p. 56). See www.playoflife.com.

This popularly written, brief book seeks to clarify the existence of "our inner gallery of roles" and to show how EMDR can heal the wounded roles or parts. She begins by describing the many aspects of roles and how our internal roles can hurt others. Roles also serve positive functions and are especially helpful when we learn to listen to them and to change their "address" from past to present. Dr. Carvalho powerfully describes the impact of trauma on our lives, and this section would be an excellent handout by itself for our clients.

Dr. Carvalho then explores Moreno's role theory. Our roles emerge in relation to those with whom we relate-parents, teachers, employers-the latter being called counter-roles. We also have psychodramatic roles in our imagination — such as our ideal mother. Having many roles facilitates our handling of many different situations in life. Those who are impoverished by having few role options do much more poorly. As we heal, we acquire new and more functional roles. Dr. Carvalho shares a striking description of a person's "emigration" from one role - a state of health-to that of another role-being ill with cancer. In the process, her reference points, friendships, and assumptions about life dramatically changed.

Engaging partial sessions of EMDR, Play of Life, and the use of both together highlight the treatment section of this book. The book closes with the summary of a moving EMDR session that successfully brought a young child role/part, an adolescent role/part, and a younger adult role/part fully into the safety of the present.

Dr. Carvalho writes simply and clearly and uses engaging metaphors and abundant quotes from her clients to illustrate her points. The translation is excellent. There are brief citations. The adaptive information processing model is mentioned and the standard EMDR protocol is used. I enjoyed this book and benefited from the integration of psychodrama and EMDR. Although it seems that this book is aimed at clients receiving role therapy who may now be getting EMDR, it speaks to a larger audience.

REVIEWED BY FARNSWORTH LOBENSTINE
Unlocking the Emotional Brain: Eliminating Symptoms at Their Roots Using Memory Reconsolidation

Bruce Ecker, Robin Ticic, and Laurel Hulley. Routledge, New York, NY, 2012, 264 pp., \$36.95 (paperback)

If you are the kind of person who balks at textbooks, you might be inclined to bypass this one. That would be a pity because this dense but not overly long book holds a cache of information relevant to eye movement desensitization and reprocessing (EMDR) in particular and to the field of psychotherapy in general about what might make certain therapies work better than others.

Authors Ecker, Ticic, and Hulley describe a therapeutic/brain process called memory reconsolidation in which disturbing memories, feelings, thoughts, and somatization are eliminated. Drawing on current neuroscientific research and rigorous clinical observation, they explain how the brain acquires memories and how unwanted reactions become established through emotional learning. To effect the lasting healing of a symptom, the brain must unlearn this ingrained information and replace it with a different truth based on contradictory current evidence.

The authors have teased out the necessary steps in this process and identified their presence in several different therapeutic approaches. Among these are their own coherence therapy, Greenberg's emotionally focused therapy, Fosha's accelerated experiential dynamic psychotherapy (AEDP), Siegel's interpersonal neurobiology, and yes, you guessed it, Shapiro's EMDR. They recognize that each approach embodies specific factors but shares common factors, too. They hold that their findings are atheoretical and offer an abundance of clinical examples from these diverse approaches to illustrate the stepwise process, including a well-documented clinical case using EMDR.

Your curiosity may now be piqued as to what the steps of this transformational sequence in memory consolidation are. I will spill the beans, but you will have to read the book to learn the proper terms and application. I take the liberty of briefly summarizing their categories.

There are three phases that involve accessing the data, reactivating the memory, activating disconfirming evidence, and repetition of these two elements. The final phase is verification that symptoms have ceased.

Sound familiar? Yes, the steps they identify do indeed coincide with EMDR's phased treatment approach, sans bilateral stimulation.

They also describe in generous detail how they conduct coherence therapy, stressing that the therapist is not limited in terms of delivering the process creatively. In most approaches, it seems to me that the onus falls on the therapist to suss out the interpretive links between the emotionally learned symptoms (target memories) and the contradictory 
evidence (e.g., positive cognitions). Then the therapist must use clinical judgment to juxtapose the contradictory truths at the right time. With EMDR, although the protocol setup initially follows a more structured framework, the therapist's stance then becomes more passive while the client is unraveling their counteractive evidence toward the great ahah!

No matter which approach, the symptom resolves itself, often in a surprisingly speedy fashion, unlocking the emotional brain and destroying the existing synaptic connection. The new learning then forms a new synaptic connection. This transformational change occurs in an experiential, not cognitive, fashion. They specifically exclude cognitive therapy as efficacious for memory reconsolidation purposes because it forms a new neural pathway of learning but does not eliminate the old memory pathway, necessitating maintenance to prevent relapses.

They also take a balanced view on the role of attachment in emotional learning and the therapeutic process, highlighting the concept of neuroplasticity (the brain's ability to change).

On the downside, the publisher's formatting is crowded and how I longed for a cartoon for light relief because there is a lot of information packed into this book! What makes it more difficult to absorb is the introduction of a new vocabulary, which occupies a six-page glossary. These terms are mostly the language of coherence therapy. Chapters repeat the main precepts: helpful for some readers and annoying to others.

In all, the authors have made a significant contribution to the field by offering a model which integrates clinical techniques with neuroscientific findings, generously verifying a range of therapeutic approaches. This in itself offers a refreshingly needed atmosphere of cohesiveness and unity in the sometimes divisive world of psychotherapy.

\section{REVIEWED BY FRANCES KLAFF}

\section{Handbook of Child Sexual Abuse: Identification, Assessment, and Treatment}

Paris Goodyear-Brown. John Wiley \& Sons, Inc., Hoboken, NJ, 2012, 612 pp., \$57.00 (hardcover)

Paris Goodyear- Brown has assembled a hefty primer on the topic of child sexual abuse (CSA). This worthwhile resource is not only beneficial for new or seasoned child therapists but also to those employed in foster care, child welfare, family law, and law enforcement.

The book details "best practices" for investigation and interventions of CSA. Ms. Goodyear- Brown divides the text into four parts: "Identification," "Assessment," "EvidenceBased Treatments and Other Effective Approaches," and "Special Issues." Each chapter within these sections is written by a leader(s) in the respective area of interest, thereby qualifying this book as a resource beyond compare.

Part 1 details the dynamics of CSA including prevalence, types of abuse, and risk factors. This section chronicles the impact of mass media, advances in identification of trauma, and the effect of CSA on brain development and health, thus provides a modern take on the dynamics of CSA.

Part 2, "Assessment," describes the role of multidisciplinary systems. Information about the use of case histories, assessments, and clinical/forensic interviewing are provided. In addition, this section establishes the value in use of evidence-based treatment and effective approaches for treatment of CSA.

These approaches are identified in the third segment with chapters written by experts on trauma-focused cognitive behavioral therapy, eye movement desensitization reprocessing (EMDR), trauma-focused integrated play therapy, parent-child interaction therapy, flexible-sequential play therapy, child-parent relationship therapy with nonoffending parents, trauma informed art therapy, group therapy and bibliotherapy. Each modality details a unique perspective for clinicians to explore.

The chapter on EMDR is written by Robbie AdlerTapia, Carolyn Settle, and Francine Shapiro who define EMDR and cite research supporting it as an effective treatment for symptoms of CSA. The authors explain the Adaptive Information Processing theory, as well as the adaptability of this theory in conceptual collaboration with other modalities. Emphasis is placed on the significant advantage of EMDR as offering a methodology to access traumatic memories without relying on the creation of a trauma narrative. Furthermore, the authors outline factors which must be considered regarding the forensic interview when working with CSA. Highlighted by the authors is the ability of EMDR to address the traumatic symptoms of the client while simultaneously creating an unimpeded investigation. Three detailed case reviews that illustrate the eight phases of EMDR are also provided.

Part 3 of the book, "Special Issues," spotlights additional considerations of CSA. Specifically, topics such as problematic sexual behavior, self-injury, adolescent populations, cultural issues, secondary trauma and vicarious traumatization, and an ecological perspective toward preventing CSA are discussed.

In summary, Ms. Goodyear-Brown has provided an advantageous resource for all who work in the challenging arena of CSA. At times redundant, this book is best suited as a clinical resource rather than a cover-to-cover read. Although previous publications have addressed the special considerations of working with child victims of sexual abuse, Ms. Goodyear-Brown offers a cohesive manual of contemporary considerations when addressing the unique needs of this population.

REVIEWED BY STACEY STEVENS 


\section{Defining and Redefining EMDR: New Clinical Strategies}

David Grand. Self-published, EMDR Brasil, Brasilia, Brasil, 2013, 106 pp., \$15.00 (paperback)

David Grand addresses eye movement desensitization and reprocessing (EMDR) clinicians and begins with an upfront admission that he is presenting his personal style as a clinician. I am someone who likes being privy to the musings and decision points of an experienced therapist. But this book is too full of extraordinary unmet clinical promises. The back cover and the table of contents list new clinical strategies involving EMDR and the treatment of trauma, ego states, and character pathology. There are sections on diagnostics, critical incidents, body processing, and performance enhancement. And the book includes questions and answers on how to use EMDR with stuttering, self-use, and fear of flying among other areas. It includes two full verbatim EMDR sessions. This would be an incredible amount of material to be covered in 1,000 pages, let alone 106 pages.

Dr. Grand's writing style is casual rather than professional, more akin to a blog than a professional publication. The book seems to have been hastily assembled without being edited or to have been converted to be sold on Amazon from a different format. There are numerous typographical errors and the table of contents inaccurately identifies page numbers as if the book were 148 pages rather than the 106 pages in the Amazon version.

My more serious concerns about the book relate to its clinical content. EMDR therapy has evolved over the years (F. Shapiro, 2001). There is a wide range of welldocumented, efficacious variations (Grant \& Threlfo, 2002; Korn \& Leeds, 2002; Luber, 2008; Popky, 2005; R. Shapiro, 2005, 2009; Jarero, Artigas \& Luber, 2011). And EMDR practitioners give cautionary warnings about the care needed when treating complex cases such as dissociatively disordered clients (Twombly, 2005). Each of the innovative contributors has delineated a rationale or a theoretical or research base for deviating from the standard, but all stay within the parameters of EMDR therapy.

Dr. Grand presents a wide range of clinical techniques but fails to explicate a coherent or cohesive theoretical foundation for his clinical strategies. He does not appear to be coming from the accepted adaptive information processing (AIP) perspective. He often asserts that continual bilateral stimulation (BLS) is more effective than the standard use of BLS/dual attention stimulation (DAS). But he does not present clinical studies or a clear rationale to validate his stance. This reviewer questions whether Dr. Grand's variations are within the EMDR parameters as currently defined by EMDR International Association (EMDRIA). Perhaps he is opening a productive debate. However, Dr. Grand fails to make a strong case for redefining EMDR therapy in this book.

David Grand is a creative and experienced clinician and one of the pioneers of EMDR therapy. His work on performance enhancement is particularly intriguing. He may be on the cusp of developing innovative and effective forms of psychotherapy. But he wraps his own personal style and clinical strategies in a cloak of EMDR and tries to reconfigure or redefine EMDR therapy to fit that style. Dr. Grand's clinical strategies as outlined in this book could mislead, distort, and set up false expectations for his readers, clinicians, and the general public alike by labeling and defining what he is doing as EMDR therapy and by oversimplifying complex clinical issues and interventions.

REVIEWED BY NATALIE S. ROBINSON

\section{References}

Grant, M., \& Threlfo, C. (2002). EMDR in the treatment of chronic pain. Journal of Clinical and Consulting Psychology, 58(12), 1-16.

Korn, D. L., \& Leeds, A. M. (2002). Preliminary evidence of efficacy for EMDR resource development and installation in the stabilization phase of treatment of complex posttraumatic stress disorder. Journal of Clinical Psychology, 58(12), 1465-1487.

Luber, M. (Ed.). (2008). Scripted protocols: Special populations. New York, NY: Springer Publishing.

Popky, A. J. (2005). DeTUR, an urge reduction protocol for addictions and dysfunctional behaviors. In R. Shapiro (Ed.), EMDR solutions: Pathways to healing. New York, NY: Norton.

Shapiro, F. (2001). Eye movement desensitization and reprocessing: Basic principles, protocols, and procedures (2nd ed.). New York, NY: Guilford Press.

Shapiro, R (Ed.). (2005). EMDR solutions: Pathways to healing. New York, NY: Norton.

Shapiro, R. (Ed.). (2009). EMDR solutions II: For depression, eating disorders, performance, and more. New York, NY: Norton.

Jarero, I., Artigas, L., \& Luber, M. (2011). The EMDR protocol for recent critical incidents: Application in a disaster mental health continuum of care context. Journal of EMDR Practice and Research, 5(3), 82-94.

Twombly, J. H. (2005). EMDR for clients with dissociative identity disorder, DDNOS, and ego states. In R. Shapiro (Ed.), EMDR solutions: Pathways to healing (pp.88-120). New York, NY: Norton.

\section{International Handbook of Workplace Trauma Support}

Rick Hughes, Andrew Kindler, and Cary L. Cooper. Wiley-Blackwell, New York, NY, 2012, 516 pp., $\$ 186.99$ (hardcover) $\$ 149.99$ (e-book)

The International Handbook of Workplace Trauma Support is intended as a guide for anticipating, planning for, and responding to various trauma experiences that occur in the workplace. Of particular value is its emphasis on the impact that traumatic events have on the health of the 
organization, from employee suicides to large-scale disasters. The emphasis on planning and triaging events is particularly well-suited for a wide community of therapists who work in employee assistance programs (EAPs) to whom the book appears to be primarily targeted.

The book primarily draws from workplace examples which have been successfully used or which were valuable lessons learned primarily in the United States and the United Kingdom, although excellent chapters exist that describe the unique cultural contexts in other countries. The breadth of workplace settings and adaptive response models which were described were a major strength for the book. Another was the level of research detail that went into some of the chapters.

Perhaps the book's strength was its greatest challenge. Because organizational structures and regional services tend to vary a great deal by country, I found myself wondering at times who the professional might be that would find the scenarios applicable for her or his work. Furthermore, I was challenged that some of the research seemed dated, including the many references to the DSM IV instead of the more recent text revision.

Whereas various topics for planning and addressing workplace trauma were instructive, I found the flow a bit disjointed at times, with chapters not always reading cohesively. As is often the case when multiple authors contribute around a common topic, there was some degree of redundancy, such as the discussions of the history of employee assistance programs (EAPs). The book was nonetheless well-edited with a keen eye toward evidence bases in the assertions that were made with respect to the various theories and models which were described.

Information about eye movement desensitization and reprocessing (EMDR) is sprinkled throughout the book, including its use for treating mild dissociative symptoms and recommended applications in several of the models which were presented. Frequent citations throughout the book segue logically into Chapter 17 , where the adaptive information processing (AIP) model and EMDR are given specific focus. This chapter, "Utilization of EMDR in the Treatment of Workplace Trauma” written by Roger Solomon and Isabel Fernandez, gives the theoretical basis for EMDR practice as well as full, step-by-step descriptions for the three-pronged and eight-phase protocols. Information is presented clearly and in straightforward fashion with the purpose of explaining EMDR to practitioners who may be unacquainted with the model. A case example is provided in which a police officer is taken through the eight phases. This brings the model to life and demonstrates its efficacy in treating one of the well-discussed workplace populations within the text. Chapter 17 was all together very strong in presenting both the AIP model and the EMDR protocols in a straightforward manner that clearly addressed the book's topic. It would have been of value perhaps to share information about EMDR International Association (EMDRIA) so that EAP therapists would have a ready reference should they elect to pursue EMDR certification.
This well-edited text presents a cohesive and easily understandable book. Authors have taken great care to explain contexts and situations, therapeutic applications, and protocols for the various methods of addressing trauma. I would highly recommend this book for any therapist who was involved or planning involvement with EAP work. It seems an indispensable guide for partnering with organizational leaders in mitigating the risk of traumatic impact on their respective work units.

\section{REVIEWED BY STACEE REICHERZER}

\section{The Rite of Return: Coming Back From Duty-Induced PTSD}

Karen Lansing. Self-published, High Ground Press, Monterey Bay, CA, 2012, 198 pp., $\$ 15.50$ (paperback)

Rite of Return: Coming Back From Duty-Induced PTSD is designed to assist first responders who need information regarding the development of acute stress and posttraumatic stress disorder (PTSD) and how to come back from the psychological injury. It provides practical guidance to this population on effective treatment options. The publication serves to educate mental health professionals with valuable information regarding the culture of warriors (law enforcement and military) and rescuers (firefighters, paramedics, lifeguards, ski patrols, and mountain search and rescue). The design of the book's chapters and the use of terminology serve to maintain a real-world authenticity in working with responders and provides a glimpse into what it is like to be a mental health professional working with them. A glossary of the terms is provided for the reader as a means of insuring everyone is on board with understanding the message of the book.

The author, an eye movement desensitization and reprocessing (EMDR) therapist, introduces the reader to the impact of PTSD among those persons who serve our nation and communities as they run toward danger during challenging times. Eight case studies are presented describing the various intense experiences from which responders developed PTSD. Readers are introduced to the efficacy of EMDR therapy in the treatment of duty-induced PTSD. The efficacy of EMDR is substantiated with full-color single-photon emission computed tomography (SPECT) imaging made at pre- and posttreatment of the responders. Of particular interest is the demonstrated change in areas of the brain such as the cingulate gyrus, the basal ganglia, the amygdala, and the cerebellum illustrated with the SPECT studies before, during, and after treatment. The evidence presented in this book offers hope to those who struggle with the disorder.

Persons who wish to understand more about the culture of first responders, their needs, and how this author has served them will benefit from reading this book. Warriors and rescuers who desire more information about

Journal of EMDR Practice and Research, Volume 8, Number 3, 2014 
duty-induced PTSD and effective treatment options will also value this book and read it intently (as will their spouses).

A strength of the book is its design for providing information to first responders and many military personnel. This book is exceptional in accomplishing this goal. An additional strength is the author's rich depth of experience in working with this population. It provides military personnel and first responders with an important understanding of stress disorders and PTSD along with options for treatment. Limitations of the book include the fact that mental health professionals will find much of the material on PTSD is familiar, even redundant. There are no new contributions toward the discussion of PTSD. However, what is added are SPECT images which demonstrate the effectiveness of EMDR therapy in the treatment of PTSD. This book makes a valuable contribution in understanding the first responder population, the impact of duty-induced PTSD, and the value of EMDR therapy as an effective treatment.

REVIEWED BY E. C. HURLEY

\section{Programs and Interventions for Maltreated Children and Families at Risk: Clinician's Guide to Evidence- Based Practice}

Allen Rubin. John Wiley \& Sons, Hoboken, NJ, 2012, 384 pp., $\$ 58.00$ (paperback)

Several years ago, noted educators Allen Rubin and David Springer set out to create a clinical series designed to keep practicing clinicians abreast of evidence-based practices. Their goal was to provide brief foundational reviews of various interventions while focusing on a "how-to" approach-enabling clinicians to immediately integrate these treatments into their clinical work.

This book is the latest installment in this series. It focuses on prevention as well as treatment, with highly specialized topics addressed. The text is divided into sections concentrating on different types of programs and treatments for high-risk children and families, including those involved with child protective services, intimate partner violence, substance abuse, and high-risk parenting practices. The section on trauma-focused interventions contains two chapters: one on trauma-focused cognitive behavioral therapy for children and one on eye movement desensitization and reprocessing (EMDR) for abused and neglected children.
The EMDR chapter, "EMDR for the Treatment of Children in the Child Welfare System Who Have Been Traumatized by Abuse and Neglect," is written by noted author and clinician Robbie Adler-Tapia, who is widely published on EMDR practices in children and adolescents. She offers a step-by-step guide for practitioners, including the basic underpinnings of both EMDR and its adaptive information processing theory as well as explaining how to adapt interventions to meet the needs of younger clients. Integrating developmental and attachment theory, Dr. Adler-Tapia offers a customized EMDR protocol by breaking down each of the eight phases of treatment into easy-to-follow steps for clinicians. It is noted that despite the inherent challenges when clients' cognitions are not fully developed, there is also a unique opportunity associated with such interventions. Dr. Adler-Tapia asserts that a key benefit to using EMDR with children is that "neurodevelopment is most rapid and malleable and treatment can have its greatest impact" (p. 142). In addition to the adapted protocol, readers are provided with an overview of considerations for the impact of child welfare involvement as well as important considerations in case conceptualization and treatment planning with this unique population. Although the chapter does not provide an illustrative case example, it does offer specific suggestions aligned with each stage of the therapeutic process. Dr. Adler-Tapia also provides the reader with references to comprehensive EMDR literature reviews.

Dr. Rubin's Programs and Interventions for Maltreated Children and Families at Risk: Clinician's Guide to EvidenceBased Practice is a selective collection of best practices for clinicians working with children and families. The chapters are authored by clinical practitioners well-known for their expertise in each of the distinctive empirically supported interventions covered in the text. In addition, Dr. Rubin provides a chapter-by-chapter summary of the empirical support for each of the interventions addressed as well as a review of the empirical support process. The latter is particularly helpful to practitioners who wish to better understand the process of clinical research as well as those who might feel motivated to contribute to the literature themselves. Content is presented in clear, easy-to-understand terms, with substantial support provided through references and suggested readings. Although targeted for therapists working with at-risk populations, practitioners in various settings will find this text to be a helpful clinical reference guide.

REVIEWED BY STEPHANIE K. SCOTT 